Logic and Logical Philosophy

Volume 15 (2006), 131-153

DOI: $10.12775 /$ LLP.2006.008

\title{
Athanassios Tzouvaras
}

\section{HOW EFFECTIVE INDEED IS PRESENT-DAY MATHEMATICS?}

\begin{abstract}
We argue that E. Wigner's well-known claim that mathematics is unreasonably effective in physics (and not in the natural sciences in general, as the title of his article suggests) is only one side of the hill. The other side is the surprising insufficiency of present-day mathematics to capture the uniformities that arise in science outside physics. We describe roughly what the situation is in the areas of (a) everyday reasoning, (b) theory of meaning and (c) vagueness. We make also the point that mathematics, as we know it today, founded on the concept of set, need not be a conceptually final and closed system, but only a stage in a developing subject.
\end{abstract}

Keywords: effectiveness of mathematics, everyday reasoning, vagueness, meaning.

\section{Introduction}

In 1960 Eugene Wigner, the distinguished physicist who three years later won the Nobel prize in physics, published an article ([19]) the title-phrase of which became ever since a popular slogan among people who had even a slight acquaintance with the epistemology and philosophy of science. Although the title of the article refers, misleadingly enough, to the effectiveness of mathematics in the natural sciences in general, its actual subject-matter is the unquestionable fact that mathematics has been the language par excellence of modern physics, a fact that has been also long ago epitomized in the phrase (attributed to Galileo) that "the book of nature is written in the language of mathematics". Wigner cites many examples to convince the 
reader about the precision of mathematical description in the formulation of the laws of physics. But when it comes to the question about the reason for that impressive effectiveness, he confines himself to saying that it is a "miracle". Moreover he closes his article as follows:

The miracle of the appropriateness of the language of mathematics for the formulation of the laws of physics is a wonderful gift which we neither understand nor deserve. We should be grateful for it and hope that it will remain valid in future research and that it will extend, for better or for worse, to our pleasure, even though also to our bafflement, to wide branches of learning.

([19, p. 9], my emphasis)

The effectiveness of mathematics in physics is just one side of the hill which, as shown from the passage cited above, sparked off and cultivated hopes that it could be extended to other parts of science and learning. The other side is that these hopes never turned into reality, and there is no sign that they will within the visible future.

The purpose of this article is twofold: On the one hand to counterbalance Wigner's thesis by pointing out important aspects of science that are (still) far from being adequately captured by the mathematical tools. And on the other to argue that this inadequacy need not be attributed to mathematics itself, but rather to its present stage of development. That is, we want to make the point that what we call today mathematics need not be the final toolkit that this art possesses for grasping reality.

Besides physics, important constituents of science are also chemistry, biology, computer science (especially its branch termed "artificial intelligence"), linguistics (as study of linguistic structures), even cognitive psychology and sociology in the wider sense of studying structures and patterns of human affairs. Nevertheless the importance of the contribution of mathematics in each of these particular sciences is evaluated as minor. This is not to be meant that mathematics do not contribute at all. What we mean is the following clearcut and striking difference that constitutes the basic fact and the main motive for this article:

BASIC FACT. While there is a great deal of important mathematics tailormade for physics, there is no important mathematics tailor-made for chemistry, biology, computer science, linguistics, commonsense reasoning etc.

Historically, there has been a very special relationship between physics and the corpus of knowledge that we call mathematics. A very fundamental part of the latter, namely the Differential and Integral Calculus, which later 
was refined, generalized, sharpened and deepened to Mathematical Analysis, was discovered by physicists for the needs of physics alone. Even today physicists regard this field as a domain ex officio "of their own", and they can hardly accept that it can have an existence independent of any application in physics. The tools of this field have been manufactured for a very specific purpose: To describe motions of points in space and time (orbits) or, in later years, behaviors of more complex dynamic systems. And saying that the tools are tailor-made for the job they were invented for is no exaggeration at all: The derivative of a function isn't just an approximate description of the magnitude that in physics is called "velocity". The derivative of a function constitutes the absolutely precise description /calculation of this magnitude. The derivative of a function $f$ is, by definition, the rate of change of a magnitude represented by $f$ that varies with time.

These tools on the other hand are hardly of key-importance for chemistry and biology, not to mention computer science and linguistics. Nor is it easy to say what tools would be of prime importance for these sciences. What is certain is that we possess nothing analogous to derivatives for the sciences in question. There has been only some new mathematics for computer science and linguistics in the framework of such independently developed branches as algebra and mathematical logic, but nothing comparable to the relationship between physics and analysis.

Why does this happen? To this question one might counter-pose another one: Why should mathematics be able to contribute significantly to each one of the knowledge fields that constitute science? Why should it be a kind of pass-key to every domain of learning? The answer to the latter question is simple: Mathematics exists (at least potentially and at least in principle) wherever there are permanent abstract patterns and structures, either static or dynamic, beneath the variety of temporal forms. It seems improbable that we can have morphological and behavioral stability without some underlying structural invariancy. The sciences other than physics investigate levels of matter organization of higher and higher complexity, as compared with the ground level of unorganized particles that physics is dealing with. One can hardly accept that there is no underlying mathematical structure, that remains unnoticed so far, in the composition of proteins, in the DNA patterns, in the flow of information, in the creation and transmission of meaning of a natural language, etc. The question is how to reveal this structure and how to represent it. Although these seem to be two separate questions, I think it is only one question with two interwoven facets. The structural uniformities in the above mentioned situations most probably pass unnoticed 
because they escape our existing mathematical means. In order to detect a structure, we must first possess a cognitive template in which to represent it. And conversely, in order to invent a new mathematical idea, we most often (though not always) have to be inspired by an external situation of things. However as a rule we tend to explain new things by the established schemes of thinking, to reduce the yet unknown to the already known. This is pretty natural: As far as we do not possess new keys, we attempt to unlock new doors by the old keys. What is not so natural is, when the old keys do not fit, to assume that no new keys are going to be available in the future for the specific doors - that no mathematics ever is going to be appropriate for the specific sciences.

But one might take a step further by asking: What are at last the limits of mathematization? Aren't there in principle any bounds to the range of learning areas in which mathematics can be fruitfully involved? This is a significant question and we must dare to bring forward a thesis even at the risk of partiality and incompleteness. To my view mathematization can be without serious loss of precision considered as a synonym of formalization, although mere formalization is only a preliminary stage and does not guarantee the development in subsequent stages of a nontrivial mathematical theory. So we shall use throughout the following hypothesis as our main implicit thesis:

Basic Hypothesis. Mathematics has a role to play in some part of knowledge if and only if the entities and their behavior involved there are formalizable, and the game of symbols thus arisen is not completely trivial. (Formal means syntactic, i.e., void of meaning and hence capable of symbolic manipulation.)

A word of caution is needed here. When we say that a part of knowledge is formalizable, we do not mean that it should be completely formalizable, i.e., all truths of the system should be captured by syntactic means. Because even the most elementary part of mathematics, that is, number theory, is not completely formalizable, as Gödel's incompleteness theorem has shown. There will always be truths about numbers that will never be decided by syntactic derivations (proofs). So a more modest formalizability requirement is set forward here: We only ask that an area of learning be formalizable up to a certain extent, but considerable enough to provide new insights and shed new light to what happens in the area in question.

So the first question remains: Why is mathematics so little or not at all effective in all science except physics? The rough explanation defended in 
this article is that the present-day mathematics lack the appropriate means to grasp and represent the uniformities outside physics. Specifically we are going to give a (necessarily incomplete) account of the insufficiency and ineffectiveness of existing mathematics in a host of interconnected fields of great interest such as (a) everyday (or commonsense) reasoning (in contrast to formal logic), (b) theory of meaning and (c) vagueness. The aforementioned areas, having as common ingredient the use of natural language, are fundamental parts of human thought and communication. Do they fulfil the assumption of the Basic Hypothesis, i.e., are they in principle formalizable aspects of reality? It is almost evident that natural language cannot be formalized in its entirety, since it is a live organism that all the time overspills the types and forms imposed from outside. But this by no means entails that many aspects that are common in all natural languages of the world, like the creation and conveyance of meaning, or the handling of vagueness, are not in principle capable of formalization. What we can take almost for sure is that the sought formalization is unattainable by the tools and methods available today.

\section{Everyday reasoning}

Reasoning in general is any mental procedure for getting (valid) conclusions out of given data. The mental procedures used in everyday life for making inferences constitute everyday reasoning. In contradistinction to everyday reasoning the term "logical reasoning" is used to entrench the kind of reasoning that obeys strict and explicit rules - rules of a well-defined system that is called "logic". For example mathematical reasoning is a prominent example of logical reasoning, since in any instance of mathematical reasoning, every step of it is the application of an axiom or rule of inference of two-valued classical logic. In a wider sense, everyday reasoning could also be thought of as a sort of logical reasoning, if one could provide a system of rules that the supposed underlying logic obeys. But in doing so one should be prepared to radically change the concept of logical axiom and logical rule as the latter are exemplified in pure logical reasoning, e.g. in ordinary two-valued formal ${ }^{1}$ logic.

\footnotetext{
${ }^{1}$ It is an old custom to call "formal"or "symbolic" the logic whose axioms can be written down in symbolic form. This however is not to be meant that any other system not so far being codified as a list of symbolic rules is in principle "informal" or "unformalizable". On the contrary, it is a main claim of this article, that aspects of reality not formalized so far, will be formalizable in the future, as a result of the enrichment of mathematics with new concepts and methods.
} 
To get an idea of the diversity of rules one should take into account, let us attempt to trace out some of the meanings (or uses) of "reasonable" (where an inference is "reasonable" if it is reached by the rules of everyday reasoning):

(a) Reasonable as natural consequence. E.g.: "It is reasonable that after such a squall of rain many houses would be flooded." Or, "Since he shot straight into the crowd it is reasonable that he would kill some body."

(b) Reasonable as fair. E.g.: "It is reasonable that after all she suffered from her husband she would like to take revenge on him."

(c) Reasonable as positively purposeful, i.e., something promoting the long-term general interest. This, in a different social code, is called "moral". E.g. Moses' Ten Commandments, are not but commandments of positive purposefulness aiming at a people's survival. "Thou shalt not kill" because if you do, others will too and in the end we shall exterminate each other. In the same spirit is Kant's Categorical Imperative: "Act only according to that maxim by which you can at the same time will that it would become a universal law." Or as Jesus has put it: "All things therefore whatsoever ye would that men should do unto you, even so do ye also unto them: for this is the law and the prophets" (Matthew 7:12).

(d) Reasonable as optimum choice. E.g.: "Since I found the same shirt cheaper in this shop, it is reasonable to buy it here."

(e) Reasonable as meaningful or justifiable. E.g.: "He suddenly left his car in the middle of the street and ran away. Quite unreasonable."

What is common in all the above meanings of the term "reasonable"? (1) They all refer to how certain (types of) facts $B$ are related to other (types of) facts $A$, which in some sense are their source or cause. For example $A=$ rain and $B=$ flood, or $A=$ bad behavior and $B=$ revenge, or $A=$ law violation and $B=$ punishment, or $A=$ immorality and $B=$ long-term decay. (2) The facts and their causal relationships belong to the specific world around us and not to some general or theoretical world. It is probable that in some other possible world rain wouldn't cause floods and bullets could pass through bodies without hurting. If I throw a stone "upward" in outer space, it is not going to fall "downward". And in some primitive societies, eating one's parents after their death is a duty rather than a crime. In short, the connections of events exhibited in an agent's everyday reasoning are mostly due to the particular physical and social order of things that happen to hold in the part of the world where the agent lives.

The specific relationships between specific types of facts constitute uniformities which are learned by an observer through experience, and to as- 
certain that a certain state of affairs is "reasonable", it only means that the events occur as they are expected to occur according to the known uniformity.

Summing up we can say that "reasonability" of everyday thinking mostly amounts to conforming with uniformities between types of events, uniformities which are due either to the laws of nature, or to laws and social conventions of humans. This is in sharp contrast to what "logical" means in the sense of formal logic.

\section{Logical reasoning and its restrictions}

As everybody knows from his/her high school days, formal logic, in its more primitive Aristotelian version, deals with syllogisms. These are inference schemes like: "All mammals are animals with lungs; some mammals live in the sea; therefore some animals living in the sea have lungs." Or "Some birds have a crest; peacock is a bird; therefore peacock has a crest". That is, every syllogism has the form "if $A$ and $B$ then $C$ ", where $A, B, C$ are declarative sentences. Some syllogisms (like the first example) are true, others (like the second example) are false. Syllogisms (more precisely true syllogisms), just like everyday reasoning, express certain permanent connections, and hence uniformities, between types of facts expressed by the sentences $A, B, C$. However, as we said in the previous section, the uniformities of everyday reasoning concern connections between specific types of events of the world around us, which happen to be in such a causal relationship to each other, because of the particular structure of the physical world, the laws of physics or the laws of human society.

In contrast to that, the connections expressed by Aristotelian syllogisms are independent of the particular physical and social order. These connections are supposed to hold in every possible situation or, as we say with a grain of salt, in every possible world. Such laws are just the logical laws. Now the fact that the syllogism is true or false independently of the particular situation that it refers to, means that a syllogism is true or false independently of the particular meaning of the terms involved, and so its truth or falsehood depends upon syntax alone. For example if in our first syllogism mentioned above we substitute the symbols $X, Y, Z$ for the terms "mammal", "animal with lungs" and "animal living in the sea", respectively, the syllogism becomes "All $X$ are $Y$; some $X$ are $Z$; therefore some $Y$ are $Z$ ". This is a valid syllogism no matter what $X, Y, Z$ denote. Indeed, the sentence "All $X$ are $Y$ " means $X \subseteq Y$, while "Some $X$ are $Z$ " means 
$X \cap Z \neq \emptyset$. The conclusion "Some $Y$ are $Z$ " means $Y \cap Z \neq \emptyset$, and this follows from $X \subseteq Y$ and $X \cap Z \neq \emptyset$ by the abstract Boolean properties of collections, which coincide with the Boolean properties of propositions.

The division of truth into contingent (of this world) and logical (of all possible worlds) is one of the most important achievements of elementary logic. At the same time it makes us realize how insufficient logical truth is to cope with the subtleties of everyday reasoning.

Logical reasoning operates effectively in a world of abstract entities and relations. An ideal such world is the universe of mathematics. The truths of mathematics are neither contingent nor logical. They belong to a third kind that Kant termed "synthetic a priori", that is, something between contingent and logical. We shall not discuss this further here since this is not our point. What is pertinent to the present issue is that the truths of mathematics are produced by a small group of initial assertions (axioms) by logical reasoning alone, i.e., by application of logical axioms and rules. Mathematical axioms are not void of meaning. They are accepted as carriers of specific mathematical content. But the face-value of this meaning is just truth and falsehood. All sentences of the mathematical language are in last analysis true or false and a logical derivation (proof) is correct if and only if each step warrants the validity of the flow of truth.

In contrast, in everyday reasoning instead of a small set of axioms, we have a huge set of initial contingent truths (a data base), concerning innumerable independent (types of) facts of the world around us (from knowledge of simple natural facts about gravity, electricity etc., to knowledge about driving, commuting in a town, cooking, child caring, etc). And instead of logical inference between assertions, we have constraints between types of events. The difference of the two systems can be highlighted by comparing their "implications" (or conditionals), i.e., the sentences of the form "if-then". Implication is the most fundamental operation of a logical system, since it realizes the one-step inferential procedure of the system. The implications of everyday reasoning are causal or conventional connections (due to physical laws, or to legal, linguistic and other constraints) of the type:

- If you fall from a height, you will be fatally injured.

- If you steal, you will be brought to justice.

- If people wear overcoats, it means that it is cold.

The general form of implications is "if $A$ then $B$ ", but (contrary to what happens in mathematical languages) not every expression of the form "if $A$ 
then $B$ " is an admissible implication. E.g. the phrases "If you steal, it means that it is cold", or "If somebody wears an overcoat, then he will be fatally injured" in plain human communication are incomprehensible. They can be used only as curious plays on words and are probably acceptable only in poetry or in some other context tolerant enough to meaning distortion.

On the other hand the implication of logical reasoning is the usual material implication denoted $A \Rightarrow B$ which makes sense for all sentences $A$, $B$. It is very well-known how far material implication is from being able to capture or comply with everyday reasoning, so there is no need to argue in length about it. It suffices only to point out that while everyday conditionals convey meaning, material implication conveys just truth. This is fairly explained by the following implication: "This letter must be sent. Therefore this letter must be sent or burned". According to commonsense reasoning the implication is obviously false. But if we write it as a material implication, i.e., in the form $A \Rightarrow A \vee B$, then it becomes a tautology. That is, if a postman burns the letters that he was given to deliver, he is in full accord with formal logic.

We said that many (though not all) conditionals of everyday reasoning express causal relationships between types of events. Let us cite one more example showing how material implication fails to capture such relationships. Consider a device consisting of a number of electric switches (say $n \geq 2$ ), serially connected to each other and to a light bulb. Obviously the bulb lights if all switches are turned on. So let $\forall s O n(s)$ denote the fact that "all switches are on", and let $L$ denote the fact that "the bulb lights". If " $\Rightarrow$ " could faithfully stand for causal relationship, then the truth of the sentence $\forall s$ On $(s) \Rightarrow L$ would represent the relationship "if all switches are on, then the bulb lights". Now the sentence $\forall s O n(s) \Rightarrow L$ is logically equivalent to $\exists s(O n(s) \Rightarrow L)$. But the latter is clearly false, since there is no particular switch whose turning on alone would cause the bulb's lighting.

Summing up: While the implication "If $A$ then $B$ " of everyday reasoning conveys meaning, the implication $A \Rightarrow B$ of logical reasoning conveys simply truth value. Truth value may be regarded as a small (and in any case not necessary) constituent of meaning. Every linguistic entity having truth value must have also meaning, but not the other way round ${ }^{2}$. In addition

\footnotetext{
${ }^{2}$ This is obvious for non-declarative sentences, such as interrogative, imperative etc., which have meaning but not truth value. But it holds also for declarative ones. The most ready to hand example is the Liar's sentence: $p:$ " $p$ is not true". The sentence $p$ has no truth value but has clearly meaning, since it "means" something: That a specific sentence is not true.
} 
truth values present some inner mathematical structure, either as a Boolean algebra or as a Heyting algebra, or some other more general structure. In contrast, at least for the time being, no mathematical structure has been possible to be built on meaning.

\section{What is meaning?}

In the preceding discussion the notion of meaning comes time and again. Meaning is like time: "For what is time? Who can easily and briefly explain it? Who can even comprehend it in thought or put the answer into words? Yet is it not true that in conversation we refer to nothing more familiarly or knowingly than time? And surely we understand it when we speak of it; we understand it also when we hear another speak of it. What, then, is time? If no one asks me, I know what it is. If I wish to explain it to him who asks me, I do not know." (Saint Augustine, Confessions, Book 11, Chapter 14.) Similarly, if one asks me, I know what meaning is. If I wish to explain it, I do not know. At least concerning time, we have constructed machines to measure it. But there is no machine so far to grasp meaning, unless (and to the extent that) the latter is reduced to syntax. This is the case of course when the language is artificial, like a programming language or a first-order mathematical language. The problem of meaning for mathematical languages is completely solved from the time of Alfred Tarski, and the theory of meaning for them is a fully-developed rich and deep branch of mathematical logic, called model theory. So the problem of meaning concerns exclusively natural language. Today this is an area lying mostly in the hands of the philosophers of language. A good list of relevant sources can be found in [3]. We are not going to enter here a discussion of the philosophical trends around this problem. We shall only touch very briefly on two aspects of the problem, that help one get an idea about its hardness. The interested reader might consult also [13] as a source book for philosophical aspects of meaning.

First, on the demarcation of meaningful from meaningless. A basic ingredient of our understanding of a concept is our ability to delimit it from its opposite, or from what it is not. For example there is a division of all phrases of a natural language into those that do have a truth value and those that do not. For some phrases the criterion is simply syntactic: For instance an interrogative phrase cannot have a truth value. For others, like the Liar's sentence (see footnote 2), the nonexistence of a truth value is proven. (Namely the assumption of the existence of a truth value leads to a 
contradiction.) Of course the division is not strictly defined ${ }^{3}$, so there can certainly be a lot of borderline instances ${ }^{4}$. But even with the existence of borderline cases, the concept of truth-vagueness is adequately demarcated.

In contradistinction there is no dependable and widely acceptable way to delimit meaningful from meaningless phrases. E.g. there is no known diagonal analogue of the Liar's sentence, which would prove the existence of meaningless (well-formed) phrases ${ }^{5}$. Of course there is a trivial method to get out of meaning: To violate grammar in an obvious and brute way, by putting words in a string at random: "cat yesterday whom fire". Linguistic entities built according to grammar are called grammatical. Random strings of words are clearly ungrammatical. But the term "phrase" (in contrast to "linguistic entity") implicitly refers to grammatical entities, so we can consider these two terms, phrase and grammatical expression, as synonymous. So the question becomes: Are there phrases (i.e., grammatical expressions) which are meaningless? Some people tend to believe (I am one of them) that meaningfulness, in the wide sense ${ }^{6}$, is identical to grammaticalness.

\footnotetext{
${ }^{3}$ In the case of a first-order language the division is strictly defined: A formula has a truth value precisely when it is a sentence, i.e., when it has no free variables.

${ }^{4}$ Many phrase instances are controversial with respect to their truth value, just as a result of the uncertainty and vagueness regarding their meaning. This is the case e.g. with counterfactuals: "If I were the present king of France, I would be bald". It is indeterminate whether it has a truth value, because its meaning (sense or reference) is obscure.

${ }^{5} \mathrm{I}$ mean a sentence $q$ the definition of which would entail that $q$ is meaningful if and only if $q$ is meaningless. Just as the Liar's $p$ entails that $p$ is true if and only if $p$ is false. Professor Buszkowski drew my attention to the sentence mentioned in [4, p. 14]: $q:=$ "This sentence is either false or fails to express any claim at all" or, equivalently, $q:=$ "If $q$ is meaningful then $q$ is false", which seems to be the meaning-theoretic analog of the Liar. The argument is as follows: If $q$ is meaningful, then $q$ is equivalent to the Liar, which is a contradiction. Hence $q$ is not meaningful. If $q$ is not meaningful, then $q$ is true. But then $q$ is meaningful (since any sentence having a truth value is meaningful). So $q$ is meaningful if and only if it is not meaningful. However I think that the first part of this argument invalidates the intended conclusion. The Liar is no more considered as a typical contradiction. Rather it is a point of departure from truth-valuedness. So the fact that $q$ is meaningful and is equivalent to the Liar constitutes no contradiction. It shows simply that $q$ is the Liar, which is a meaningful sentence without truth-value.

${ }^{6}$ Meaning being a vague and conditional (i.e. contextual) notion, the question of meaningfulness in not a yes/no question. It admits various degrees of clarity, as well as borderline cases. There is straightforward meaning conveyed in mainstream conversation, but there is also meaning undercover or in disguise or ambiguous, expressed in special occasions. For example the implication "If you steal, it means that it is cold", mentioned in the previous section, lacks straightforward meaning indeed, but it might be assigned meaning under special circumstances that one could easily manufacture. This implication is an
} 
Noam Chomsky [8] rejects that. He says that on the one hand there are ungrammatical meaningful expressions, such as

(a) "Read you a book on modern music?",

(b) "The child seems sleeping", ${ }^{7}$

and on the other there are grammatical and yet meaningless expressions such as

(c) "Colorless green ideas sleep furiously".

However to my view, (a) and (b) stand only as imperfect or awkward substitutes for their fully grammatical counterparts. As far as one can almost immediately restore the disabled phrase, and hence its meaning, then the disabled phrase is "essentially grammatical". On the other hand it is at least doubtful whether (c) is meaningless. If we reject it, we should also reject a vast amount of past, present and future literature as meaningless, e.g. the totality of surrealistic poetry and not only that.

A second aspect that makes meaning extremely difficult to formalize is the notoriously decisive role of context. The same phrase, e.g. "you are a thief", is dressed on quite different meanings when uttered by different people and addressed to different people in different situations. Context is a semantical domain that acts like a filter or transformer that shapes the ultimate form of meaning of an utterance when the latter passes trough it. This is nicely illustrated e.g. by Stanisław Ulam in a conversation with Gian-Carlo Rota:

Now look at that man passing by in a car. How do you tell that it is not just a man you are seeing, but a passenger? When you write down precise definitions for these words, you discover that what you describe is not an object, but a function, a role that is inextricably tied to some context. Take away the context, and the meaning also disappears. [...] What is it that you see when you see? You see an object as a key, you see a man in a car as a passenger, you see some sheets of paper as a book. It is the word 'as' that must be mathematically formalized,

example of a phrase that is meaningful "in the wide sense". I believe that all grammatical phrases could be assigned meaning under very special circumstances, that's why they can be classified as potentially meaningful.

${ }^{7}$ The expressions follow the syntactic pattern of the grammatical analogues

$\left(a^{\prime}\right)$ "Have you a book on modern music?",

$\left(b^{\prime}\right)$ "The book seems interesting", respectively. 
on a par with the connectives 'and', 'or', 'implies' and 'not' that have already been acceptable into a formal logic. Until you do that, you will not get very far with your AI problem.

[15, pp. 98-99]

What can mathematics say about the problems cited above, especially the contextual character of meaning? As far as I know, the only serious, systematic and lasting project for a mathematical theory of meaning has been undertaken by Jon Barwise, in the early eighties. Of course this is not to be meant that there have not been proposed other theories of meaning before. The emphasis is on "mathematical". While other theories had used one or the other kind of soft logical and/or mathematical formalism (e.g. Frege [11] (meaning and denotation), Ajdukiewicz [2] (meaning postulates of language), Carnap [7] (meaning as the method of verification), intuitionists [12] (meaning as proof), Montague [14] (meaning as intension)), Barwise' theory was from the outset intended to create new powerful mathematical tools and prove hard mathematical theorems. However the plan was not accomplished (see below). As Professor Buszkowski remarked "the weakness of Barwise's approach was his overestimation of set-theoretic methods and at the same time an underestimation of other logical issues (nonclassical logics, modal logics, intensional logics etc)." I agree with the first part of this comment, but not with the second part, since all methods used in mathematics today are, at last analysis, set-theoretical! The methods of nonclassical logics, modal logics, etc., are not but special techniques easily representable by setstructures (for example the net mathematical objects behind possible world semantics used in modal and intensional logics are just sets equipped with binary relations). Barwise believed, at least in the first stages of his endeavor, that the ultimate mathematical notion of set would be proved adequate even for the new task of capturing and representing the extremely intricate and elusive notion of meaning. For that purpose he didn't hesitate to go beyond ZFC, into the controversial realm of non-well-founded sets as formalized by P. Aczel [1], in order to cope with circular phenomena occurring in thinking and more generally in intensional situations (see [4] and [5]).

The project started with the publication of [6] and led gradually to the so called "situation logic" and "situation theory". ("Situation" here is a coding name for the key-factor of context.) J. Barwise was already a wellknown logician (see e.g. Barwise compactness theorem), when he decided to make the crucial step from the semantics of mathematical language to the semantics of natural language and thus to the theory of meaning. The Center for the Study of Language and Information (CSLI) that was founded 
in 1983 for this purpose, produced and is still producing a large number of publications relevant to the subject. In 1989 CSLI published Barwise's book [3] that contains an anthology of articles by the author, and at the same time is a kind of synopsis that epitomizes and assesses the research done over the past 10 years in situation theory. In order to reinforce my argument that theory of meaning have always been among the pursuits of mathematics, but the tools for attain it have been and still are inadequate, I shall quote a few passages from [3]. In the end of the book we read:

The dream of a mathematics of meaning has inspired mathematicians from Aristotle, to Descartes, on to Peirce, Frege, Russell and more recent logicians. It has gotten logic where it is today. What I am saying is that we must not rest content with the current state of things, but we must pursue the dream that got us this far.

A mathematics of meaning, when it really comes of age, will have profound implications for a host of problems that bedevil us today, problems in the study of language, intelligence and the mind. Situation theory is one attempt to develop such a field. Naturally enough, I think it is headed in the right direction. But whether or not that is the case is less important than the vision that a calculus of meaning is a possibility, a challenge worth the effort.

[3, pp. 295-297]

A little later, when the author evaluates the new mathematical tools that had been used in the development of the theory, the optimistic message turns into melancholy:

But mixed with these feelings of the importance of the enterprize, and of progress we have made carrying it out, are competing feelings. For I have to face the fact that we have failed, so far, to turn the theory into a piece of serious mathematics. Worse, having these papers all before me at once makes me realize what a small fraction of my own research over this decade has been spent in contributing to the mathematics which is my real dream.

But he soon regains his optimism:

I still think that situation theory is on the right track to a mathematics of meaning. Of course, I might be wrong. Maybe some other approach entirely [different] will emerge. But of one thing I am sure: Someone will eventually lay the foundations of a theory of meaning.

Barwise closes the book with the following fragment of the dialogue between Stanisław Ulam and Gian-Carlo Rota, part of which we have already mentioned above:

- Rota: But if what you say is right, what becomes of objectivity, an idea that is so definitely formalized by mathematical logic and the 
theory of sets, on which you yourself have worked for many years of your youth?

- Ulam: Really? What makes you think that mathematical logic corresponds to the way we think? You are suffering from what the French call deformation professionnelle...

- Rota (in fake amazement): Do you then propose that we give up mathematical logic?

- Ulam: Quite the opposite. Logic formalizes only very few of the processes by which we actually think. Time has come to enrich formal logic by adding to it some other fundamental notions [...]. Do not lose

your faith. A mighty fortress is mathematics. Mathematics will rise to the challenge. It always has.

\section{Mathematics and vagueness}

Behind the inability of existing mathematics to cope with natural language and meaning lies, to my view, its inability to cope with something more fundamental, something that infiltrates entirely natural language and meaning, namely vagueness. The vast majority of nouns and adjectives of a natural language, expressing objects and properties, respectively, are vague. The term "vague property" means here a property whose extension has no crisp boundary, but rather its boundary is a grey region. For example properties such as "tall", "smart", "bald", "blond", "clear", etc., as well as notions such as "tree", "lemon", "fish" etc., are all of this kind. Examples of crisp properties are "dead", "pregnant" and of course the mathematical entities and their properties, such as "even number", "prime number", "continuous function" etc. If we identify a notion with its extension, i.e., a collection $X$, then the vagueness of $X$ consists in that there are objects which definitely belong to $X$, others that definitely do not belong to $X$, and others that it is difficult to tell whether they belong to $X$ or not (or they belong up to a certain degree). One of the least comprehensible facts is how human communication flows unhampered in a language governed by the rule of vagueness! Meaning itself is a highly vague concept, with a thick grey zone between meaningful and meaningless.

What can mathematics do toward grasping vagueness? Some may say that every endeavor is doomed to fail before it starts, because mathematics, being the archetype of clarity and precision, cannot in principle represent right its opposite. But this is a shallow and superficial argument. The real question is whether vagueness has some structure and presents some uniformities. 
Mathematics found already by the early sixties a method to represent and handle in a practical level some aspects of vagueness. I refer to the L. Zadeh's theory of fuzzy sets (see [20]). Let $V$ be a class of objects. A crisp subset $X$ of $V$ can be thought of as a function $X: V \rightarrow\{0,1\}:$ For every $x \in V, X(1)=1$, if $x \in X$, and $X(x)=0$, if $x \notin X$. Now if $X$ is vague and there are grey regions along its boundary, it suffices to replace the two-element set $\{0,1\}$ with the real interval [0,1], and call fuzzy subset of $V$ every mapping $X: V \rightarrow[0,1]$. If for instance $X(x)=.85$, then we say that " $x$ belongs to $X$ with degree .85 ". With a little care we can extend many of the operations and relations between ordinary sets to fuzzy ones. E.g. for the complement of $X$ we define $(V-X)(x)=1-X(x)$, while $X \subseteq Y$ if $X(x) \leqslant Y(x)$, for all $x \in V$.

But such a "quantification" of vagueness, though it can find useful applications to modern technology (some washing machines use already programs in fuzzy logic in order to regulate the supply of cold and hot water) is far from helping us understand the inner structure of the situation. Does vagueness have a structure? We guess yes, and some of its aspects are apparent. Vague predicates (just like crisp ones) partition reality (more precisely a certain piece of it) into disjoint parts. The difference (compared to crisp predicates) lies in the boundaries. E.g. The predicate "even" partitions natural numbers into two classes, - evens and odds - whose boundary has null breadth. The passage from one class to the other constitutes a jumpnothing lies between. In contrast, the predicate "hot" partitions the various states of water into two classes again - hot and not hot-but now the passage from one class to the other is made through a long hazy borderline region, and the passage itself is not quite comprehensible. How (or when) exactly do we pass from the state of non-hot to the state of hot water, if we add to the cold water a unit of hot water per unit of time? This is the same type of situation as the one more vividly illustrated in the so called Bald Man (or Sorites) Paradox: If a man loses a single hair from his head each day, then surely one day he will be bald. But there is no definite such day. Because it is agreed that if we remove a single hair from a non-bald man, he remains non-bald (and equivalently, if we add a single hair to a bald man, he remains bald). It is clear that in this form vagueness is a kind of failure of mathematical induction.

The aforementioned passage from non-hot to hot water cannot be represented with conventional quantitative means. Indeed, suppose we assign numbers from $[0,1]$ to the scale of cold/hot states of water, using e.g. a thermometer. Then each vague property describing a state of the water 
temperature (e.g. "cold", "cool, "very warm" etc.) reasonably corresponds to an interval of $[0,1]$. Moreover incompatible states (like "cold" and "hot") correspond to disjoint intervals. The vague predicates hot and non-hot are complementary, so they correspond to intervals $I_{h}$ and $I_{n h}$ of $[0,1]$ such that $I_{h} \cap I_{n h}=\emptyset$ and $I_{h} \cup I_{n h}=[0,1]$. Note that $I_{h}$ and $I_{n h}$ need not be the two halves of $[0,1]$, i.e., $I_{n} h=[0,0.5\rangle$ and $I_{h}=\langle 0.5,1]$ (where $\rangle$ is ) or $]$ ). It may well be for instance that $I_{n} h=[0,0.7\rangle$ and $I_{h}=\langle 0.7,1]$. However, whatever the form of these intervals could be, for every $\varepsilon>0$, we can find a non-hot point $N$ and a hot point $H$, such that the distance $|N H|$ is less than $\varepsilon$.

On the other hand, if the above attribution of numbers represents faithfully the situation, any two hot points or any two non-hot points should stand closer together than any two points of which one is hot and the other non-hot. That is, if the representation were faithful, we should have $\left|N_{1} N_{2}\right|<\left|N_{i} H_{j}\right|$ and $\left|H_{1} H_{2}\right|<\left|N_{i} H_{j}\right|$ for any two hot points $H_{1}, H_{2}$ and any two non-hot points $N_{1}, N_{2}$. But this contradicts the fact that hot and non-hot points can be arbitrarily close together. There is some mathematical representation that conforms to the above commonsense requirement, but it requires the use of nonstandard natural numbers ${ }^{8}$, which however have no application at all in real situations (see [17]). The problem becomes acuter when instead of two-class partitions of reality (generated by a single predicate) we consider many-class (vague) partitions produced by natural kinds or color shades. Such partitions have potentially infinitely many classes, since between any two species, or colors, there is potentially a third one. Two rose objects or two red objects should be less far apart than one rose and one red object. In addition, between rose and red it should always be room for a third subtler shade to be interpolated (this is a "density requirement"). Usual real-valued metrics cannot represent partitions of this kind for the same reasons as before. But the nonstandard-valued metrics of [17] may capture some of these structural aspects of vagueness ${ }^{9}$.

\footnotetext{
${ }^{8}$ Briefly, instead of the real interval [0,1], one may consider a nonstandard model $M$ of arithmetic. For $I_{n h}$ we can take a cut $I$ of $M$ closed with respect to addition, i.e., $a, b \in I \Rightarrow a+b \in I$. Then for every $a, b \in I$ and for every $c \in M-I,|a-b|<|a-c|$, which partially fulfills the above requirement.

${ }^{9}$ Recently I observed that one can obtain appropriate modelings of vague partitions, if one changes the underlying metric of the space instead of the numbers employed. Let $(X, d)$ be an ordinary metric space. A partition $\left\{X_{i}: i \in I\right\}$ of $X$, finite or infinite, is said to be nice if for all $i \neq j$ and any $x, x^{\prime} \in X_{i}$ and $y, y^{\prime} \in X_{j}, d(x, y)<d\left(x, x^{\prime}\right)$ and $d(x, y)<d\left(y, y^{\prime}\right)$. A nice partition is exactly what one needs (except of the density requirement) for the representation of vague partitions of reality. None of the ordinary metrics (e.g. the Euclidean metrics on $\mathbb{R}^{n}$ ) allows for the existence of nice partitions.
} 
Everybody knows however that in dealing with all these vague predicates our mind makes no use of real numbers, nonstandard numbers, or any other mathematical device; moreover it does not leave the ground of two-valued classical logic either, for the sake of a supposedly more flexible many-valued "fuzzy logic". How does it succeed then in being so effective? A partial answer is that natural language is incredibly flexible. It includes structural rather than quantitative mechanisms, namely intensity determiners - such as "much", "very", "very-very", "a little" etc - as well as comparatives such as "taller than", "much better than" etc. Moreover, it is an implicit rule built in the use of language, that only actually vague predicates admit intensity determiners, not crisp ones. (That is why the phrase, say, "a little pregnant" sounds bizarre or just funny.) From a mathematical point of view the intensity determiners are operators. Actually they can be reduced to a single operator $v$ (for very). If $X$ is, say, the class of tall men, then $v(X)$ is the set of very tall men, $v^{2}(X)$ is the set of very tall men etc. We postulate that for every $X, v(X) \subseteq X$ and thus $X \supseteq v(X) \supseteq v^{2}(X) \supseteq \cdots$. Endowed with appropriate axioms, $v$ can represent many important aspects of vagueness and, interestingly enough, can also recover (up to a certain extend) Zadeh's real number approach. Such a structural approach to vagueness is contained in [18].

Despite the above isolated approaches to various aspects of vagueness, the phenomenon itself remains elusive. The available mathematical means seem inadequate. A "calculus of vague predicates" (I mean an authentic one, not simply a simulating caricature) does not seem attainable for the time being - just as a calculus of meaning, as envisaged by Barwise, does not seem attainable either.

\section{The set-theoretic ontology and Castoriadis" "magma"}

The preceding discussion might bring forward once again the question "what is mathematics'? Asking that, one is interested not in what the "nature" of mathematical entities actually is, but rather in the closeness or openness of

So, are there nice partitions at all? The answer is yes. Here is a simple example: Let $(X, d)$ be a metric space, and let $X_{1}, \ldots, X_{n}$ be pairwise disjoint and bounded subsets of $X$, with bounds, say, $M_{1}, \ldots, M_{n}$ respectively. Let $Y=X_{1} \cup \cdots \cup X_{n}$. Pick some real $M>M_{1}, \ldots, M_{n}$ and define $\rho: Y^{2} \rightarrow \mathbb{R}$ as follows:

$$
\rho(x, y)=\left\{\begin{array}{l}
d(x, y), \text { if } x, y \in X_{i} \text { for some } i, \\
M, \text { if } x \in X_{i} \& y \in Y_{j} \& i \neq j .
\end{array}\right.
$$

It is easy to see that $\rho$ is a metric on $Y$ and $\left\{X_{1}, \ldots, X_{n}\right\}$ is a nice partition of $(Y, \rho)$. 
the mathematical universe. Is mathematics a more or less completed system, ultimately defined as the universe of ZFC (or some variant of it), where of course new, undefined today, entities may appear in the future, but where all entities will eventually be sets or classes? To put it somewhat differently: Is an abstract entity mathematical if and only if it has a set-theoretic representation? To be honest, for the vast majority of the mathematicians of today the answer is yes. In particular this is the dominant view among researchers who shape the face of science at the given moment. We can hardly think that we can go somewhere beyond sets ${ }^{10}$. Set has been declared the ultimate, irreducible mathematical entity. Its simple figure has been deeply engraved into every mathematician's intuition, thought and language. Any attempt to formalize and represent some aspect of the reality, whatever, starts with the invocation of a "set". And once this is done the subsequent steps are almost expected: The set is going to be endowed with some relations, either orderings or equivalences, maybe some topology, and then we are going to prove some facts or to appeal to known theorems etc, etc.

I wonder if the notion of set has gradually and unnoticeably been grown into an obstacle and a barrier for our thought and imagination. Lest should we break the barrier in order for mathematical thinking to reach new presently inconceivable territories? Meaning, for instance, is not, nor seems capable of being, representable by sets. Nor a "vague set" is a set. Nor language - the language used by a concrete user (the "internal" language of Chomsky, in contrast to the "external" language of Montague, see [16]) - is a set. Nor Castoriadis" "magma" is a set or even a class.

Cornelius Castoriadis ${ }^{11}$ in his seminal book [9] embarks on a critical analysis of what he calls "identistic- setistic" (identitaire-ensembliste) logic.

For twenty five centuries, the Greek-western thought is being developed, expanded and refined upon the following thesis: To be means to

\footnotetext{
${ }^{10}$ Of course we do not ignore the alternative approach to foundations based on the notion of category. But from the point of view of this article the category and set-theoretic approaches are rather equivalent. Clearly categories encompass sets as a particular "topos". And conversely: If a situation is representable by means of category-theoretic tools, these tools, being essentially functions, can in turn be copied in the usual set-theoretic universe, and so the situation is also representable by sets.

${ }^{11}$ Cornelius Castoriadis (1922-1997) has been a prominent social and political thinker of our time. He spent most of his life in Paris. From 1979 till his death he was Director of Studies at the École des Hautes Études en Sciences Sociales (EHESS). [9] is widely considered his main work. Although a humanitarian philosopher by training, he had got an impressive and solid background in science, especially in economics, mathematics and theoretical physics.
} 
be something determined, and to say means to say something determined; and of course, to say the truth means to determine the saying and what is said in terms of the being or to determine the being in terms of the saying, and the eventual verification of the congruence of these two. This result which relies on the demands of a certain aspect of saying, was neither accidental nor indispensable; it was the proclaiming of Logos (ratio) as the institutionalized thought by the West. [...] I call the logic mentioned above identistic (identitaire), as well as setistic (ensembliste), being aware of the terminological abuse of language, for reasons which will be clear immediately. [...] The most advanced and wealthy reach of the identistic logic is the working out of mathematics. [...] We are interested here in the elementary logical principles of set theory, because these principles abbreviate, clarify and point out vividly what was always subject to this logic.

([9, p. 319-321], my translation)

Castoriadis proposes the verb "setify" (ensembliser) to signify the act of "creating sets" out of something pre-existent and rather undifferentiated. This kind of undifferentiated reality out of which the setistic-identistic logic creates sets, classes, objects and properties, is, roughly, what he calls magma.

We aim at revealing the way of being of what is given, before the human mind imposes upon it the setistic-identistic logic; this, which is offered in that way of being, is what we call magma. Obviously, giving a typical definition of the notion, is out of question. Nevertheless the following statement might not be quite useless:

Magma is that from which we can extract (or in which we can construct) setistic organizations in indefinite number, but which can never be recovered (ideally) by a finite or infinite combination of any of these organizations.

[9, p. 479]

Objects and properties of the ordinary world seem to be the outcome of the ability of our mind for separation, partitioning and individuation. Sets and classes are also products of this very mental mechanism. Castoriadis, mentions times and again Cantor's well-known "definition" of set: "A set is a collection into a whole of definite and separate objects of our intuition or our thought. These objects are called 'elements' of the set." Any specification of a set is clearly an act of separation and individuation. When we say "let $X$ be the set of all $x$ such that [...]", we focus on a particular part of reality, we individualize it and cut it off as a separate object by an act of saying, i.e., a linguistic term (a formula).

On the other hand, the main characteristic of magma, that distinguishes it from the ordinary sets and classes, seems to be the fact that its "ele- 
ments" are neither fully determined nor fully distinguishable and separable from each other. As a consequence, we can't construct new magmas or submagmas by the acts of saying, i.e., by the help of linguistic properties.

As a magma the (collection of) meanings of the language are not elements of a set that are subject to determinacy as a condition and manner of being. A meaning is unboundedly determinable, without being determined. It can always be pointed out, be temporarily assigned an identistic element (as in naming), be 'something' as a departure in an open series of successive determinations. But these determinations can never completely exhaust it. Moreover, they can enforce us, and actually they always do it, to return to the 'something' of the departure and consider it as 'something else'.

[9, p. 483]

In [10] Castoriadis devotes a whole chapter to the subject entitled "The logic of magmas and the problem of autonomy". There he attempts to axiomatize magmas by the help of four axioms. The axioms are formulated in the natural language using a rather obscure primitive relation of signifying, or pointing out (repérage). Unfortunately, as he himself shows, the axioms in question are contradictory. (See [10], p. 299 ff.)

\section{Epilogue}

We shall close by returning to Wigner's article referred to in the beginning. The author also poses, as we did, the question "what is mathematics?" and answers as follows:

Somebody once said that philosophy is the misuse of terminology which was invented just for this purpose. In the same vein, I would say that mathematics is the science of skilful operations with concepts and rules invented just for this purpose. The principal emphasis is on the invention of concepts.

[19, p. 2]

And he adds a little later:

Most more advanced mathematical concepts, such as complex numbers, algebras, linear operators, Borel sets - and this list could be continued almost indefinitely - were so devised that they are apt subjects on which the mathematician can demonstrate his ingenuity and sense of formal beauty.

Actually this is a good enough definition, because it refrains from speaking about mathematical ontology, and thus it may embrace both what mathematics is today and what is going to be in the future. It focuses only on 
"skilful operations with concepts and rules" without putting any restrictions to the sort of these concepts and rules. There is an objection, however. It concerns the phrase "invented just for this purpose". I do not think that, as a rule, one does mathematics just in order to play skilfully with concepts and operations. If that were the case, then chess would be a prominent branch of mathematics, but it is not. Why? Because mathematical activity ought to be meaningful, i.e., although it sometimes presents itself as a light and leisurely play, its true source and origin is eventually the outer world (as well as the inner world of our consciousness as an objectified reality), not just the playful dispositions of a boring mind. So in conclusion, to relate the main idea of this article with Wigner's definition, we need to invent new mathematical concepts and skilful operations, even of the most radical sort, in order to make some as yet formally unrepresentable aspects of reality, representable and comprehensible.

Acknowledgements. I would like to thank Professor Wojciech Buszkowski for reading an earlier draft of this paper and making useful remarks and suggestions. I also thank my friend and colleague Aristides Gogoussis who offered valuable comments that improved significantly the presentation of the paper.

\section{References}

[1] Aczel, P., Non-well-founded sets, CSLI Lecture Notes, No 14, 1988.

[2] Ajdukiewicz, K., Pragmatic logic, D. Reidel P.C. 1974.

[3] Barwise, J., The situation in logic, CSLI Lecture Notes, No 17, CSLI Publications, Stanford 1989.

[4] Barwise, J., and J. Etchemedy, The Liar. An essay on truth and circularity, Oxford University Press 1989.

[5] Barwise, J., and L. Moss, Vicious circles: on the mathematics of circular phenomena, CSLI Lecture Notes 1996.

[6] Barwise, J., and J. Perry, Situations and attitudes, Cambridge, MA, MIT Press, 1983.

[7] Carnap, R., Meaning and necessity, Univ. of Chicago Press, 1956.

[8] Chomsky, N., Syntactic structures, Mouton, Hague 1957.

[9] Castoriadis, C., L' institution imaginaire de la société, Editions du Seuil, Paris 1975. 
[10] Castoriadis, C., Domaines de l' homme, Editions du Seuil, Paris 1986.

[11] Frege, G., "On sense and reference", in: D. Davidson and G. Harman (eds.), The logic of grammar, Dickenson, Encino, California 1975, pp. 116-128.

[12] Heyting, A., Intuitionism: An introduction, North Holland, Amsterdam 1956.

[13] Marciszewski, W., (ed.), Dictionary of logic as applied in the study of language. Concepts, methods, theories, Martinus Nijhoff Publishers 1981.

[14] Montague, R., Formal Philosophy: selected papers of Richard Montague, R. H. Thomason (ed.), Yale U.P., 1974.

[15] Rota, G.-C., "The barrier of meaning", Letters in Math. Physics 10 (1985), 97-99.

[16] Smith, N., Noam Chomsky - Ideas and ideals, Cambridge Univ. Press, 1999.

[17] Tzouvaras, A., "Modeling vagueness by nonstandardness", Intern. J. of Fuzzy Sets and Systems 94 (1998), 385-396.

[18] Tzouvaras, A., "An axiomatization of "very" within systems of set theory", Studia Logica 73 (2003), 413-430.

[19] Wigner, E., "The unreasonable effectiveness of mathematics in the natural sciences", Communications in Pure and Applied Mathematics 13 (1960). Available also in URL: http://nedwww.ipac.caltech.edu/level5/March02/Wigner/Wigner.html

[20] Zimmermann, H.-J., Fuzzy set theory and its applications, 2nd Edition, Kluwer Academic Publishers, 1991.

Athanassios Tzouvaras

Aristotle University of Thessaloniki

Department of Mathematics

54124 Thessaloniki, Greece

e-mail:tzouvara@math.auth.gr 